\title{
The influence of cataractus vision in the individual characteristics of handwriting and the implications in the work of forensic document examiner
}

\author{
Michail PeRTSINAKIS
}

Chartoularios Ltd. Laboratory of Questioned Document Studies, Athens, Greece

\section{Introduction}

The literature shows that there is no work in the field of forensic document examination and the graphonomics regarding the influence of cataractus vision in the individual characteristics of handwriting. This is something that could be perceived as peculiar due to how common this disease is, especially to the population group of older people (Hodge et al., 1995), upon which the FDEs highly focus. Based on the above, this research aims to: a) show whether cataractus vision has no effect on the static individual characteristics of handwriting (null hypothesis) or that it changes it to a significant degree (alternate hypothesis), b) to pinpoint the individual characteristics that are affected by this disease and could be noted as signs of limited visual feedback and c) to investigate whether the absence of visual feedback could jeopardize the results of the comparison done by an FDE and lead to an erroneous conclusion. 


\section{The Nature of cataracts}

The healthy crystalline lens is a transparent, biconvex structure. Its primary function is to transmit the incident light and to focus it on the retina, providing the eye with a focusing refracting power of $20+$ diopters [diopter is the unit of measurement of the optical power of a lens, that is of the degree to which a lens converges or diverges light] (Slamovits, 1993). This requires that the lens retains its transparency, a condition dependent on the structural organization between the constituent proteins and water. Figure 1 presents a pictorial description of the healthy crystalline lens in the human eye.

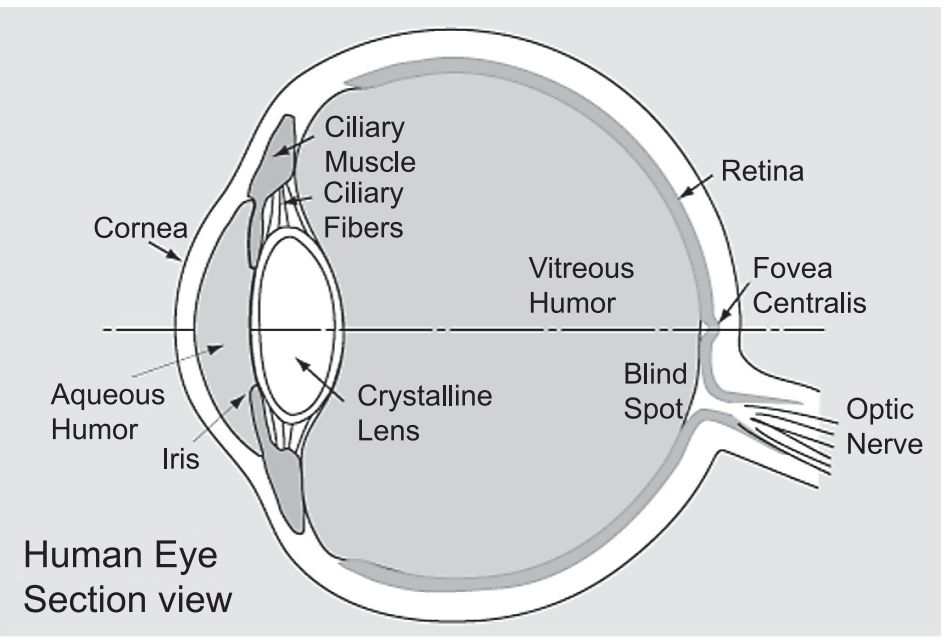

Figure 1. The position of the crystaline lens in the human eye

Cataract is a common disease of the crystalline lens, a vision-impairing disease characterized by gradual, progressive thickening of the lens, creating a clouding effect varying in degree from slight to complete opacity, thus obstructing the passage of light (Ocampo, 2000). Cataracts occur when the lens loses its transparency by either scattering or absorbing light such that visual performance, assessed through functional visual acuity recording, is compromised (Yamaguchi et al., 2011). In Figure 2 the magnified view of the cataract, under slit-lamp examination, is seen. The opaque lens is clearly seen at the centre of the eye. 


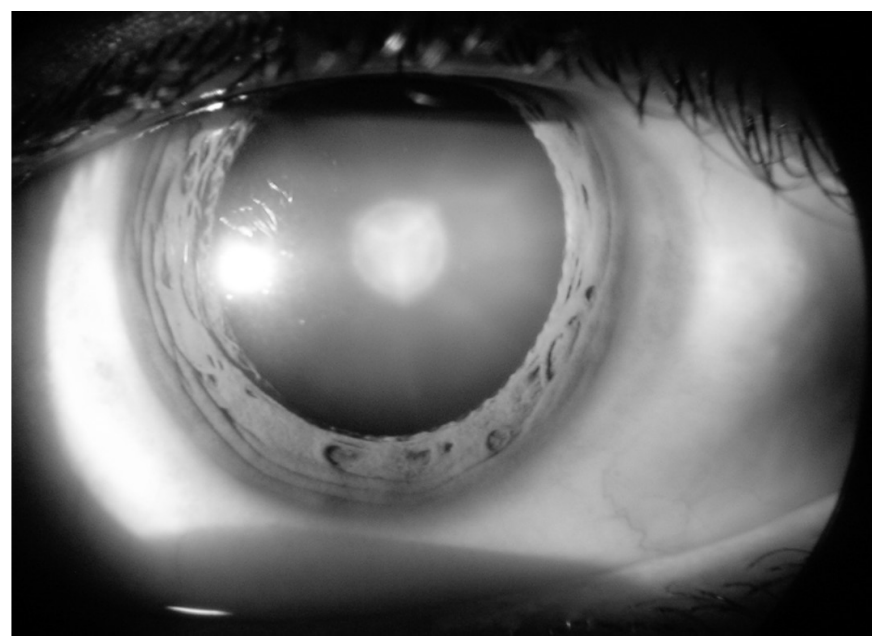

Figure 2. Opacification in the centre of crystalline lens caused by cataract

By far the most important risk factor in the development of cataracts is age - age related cataracts constitute the great majority of all cataracts (e.g. congenital and juvenile cataracts are relatively rare) and are a major public health problem in the world (Hodge et al., 1995). In developing countries, where the availability of surgical facilities is limited, age-related cataracts are the leading cause of blindness (Pavan-Langston,1990). In fact, age-related cataracts are responsible for $48 \%$ of world blindness (approximately 18 million people) (Livingston, Carson, Taylor, 1995).

Oxidative damage to lens constituents is believed to be a primary factor in the formation of age-related cataracts (Beebe et al., 2010). Studies show that oxidative stress can be cataractogenetic, since eye exposure to $\mathrm{x}$ rays or to high levels of other types of radiation, including ultraviolet (UV), can cause the development of cataracts with definite oxidative effects in the lens (Duker et al., 2008). Further support for this hypothesis comes from epidemiological studies that have found an association between increased exposure to sunlight and ageing-related cataract (Sliney, 1994).

As a result of the cataract, the quality of visual performance is compromised for both distance and near vision. The effect of cataract on functional impairment will vary depending on the type of cataract, its location and the degree of opacification. For example, since posterior 
subcapsular cataracts are usually located in the center of the lense, result in an increase of light scattering and of interference with the ability of the eye to focus an image on the retina, leading to significant subjective symptoms in its early stages. Based on the degree of visual impairment all types of cataracts are usually classified from Mild (Grade 1) to Severe (Grade 5) (Yanoff \& Duker, 2008).

No pharmaceutical treatment which can inhibit, postpone or reverse a cataract has been found, leaving surgery as the only viable solution (Kador, 1983). Cataract surgery is one of the most commonly performed surgical procedures in Europe with extremely rare complications (Mojon-Azzi and Mojon, 2007). Until the early 1980s the preferred method of cataract surgery was intra-capsular extraction (ICE) (Meadow, 2005). At that point the most common surgical option is the removal of the opaque lens and its replacement by an artificial intra-ocular lens (IOL) (Slamovits, 1993). Complications after cataract surgery are relatively uncommon and variable and may appear during the surgery, the immediate or the later post-surgical phase (Jaffe, 1989). However, in the presence of residual post-operative refractive error, the use of spectacles correction for distance may be needed (Abdelghany \& Alio, 2014). Residual refractive error may be due to planned or unexpected under-correction or over-correction by the IOL power and/or due to pre-existing corneal astigmatism or induced corneal astigmatism caused by saturation of the incision (Slamovits, 1993). The postoperative recovery period is usually short (Cunningham \& Riordan-Eva, 2011). Cataract surgery, even in cases when both eyes are similarly affected, is usually performed first on one eye and then on the other, in order to avoid any devastating complications, i.e. binocular postoperative endophthalmitis, but also other errors, for example post-operative refractive error due to inaccurate biometric assessment, however, simultaneous bilateral cataract surgery is gaining in popularity worldwide, since it has certain advantages, such as the reduction in medical visits (important in cases of older patients with other health problems), avoidance of inter-procedural anisometropia and decreased stereopsis, and very rapid rehabilitation making the surgery much easier on the patients and their families (Smith \& Liu, 2001). 


\section{Methodology}

Volunteers were recruited from patients attending for intraocular cataract removal and lithotripsy at University Hospital of Heraklion, Crete, and screened to ensure conformity to the eligibility criteria. They provided samples of their handwriting and signature before surgery (Phase A) and 3 months after during routine post-operative visits to the hospital (Phase B). The pre-surgical handwriting and signature samples of each patient were compared to the post-surgical samples of the same patient, as the vision is back to its pre-cataract healthy status at that time. The approval of the pertinent Ethics Committee was granted for this research.

The group of the pre-surgical samples of a patient was examined and the extent of their natural variations was noted, accordingly to the methodology of forensic document examination. The same procedure took place in the post-operative samples of the same patient. These two groups were afterwards compared to each other, focusing mainly on the individual characteristics of general design, line quality, horizontal and vertical extensions, inter-allograph ration and spacing. The result of the comparison of each characteristic showed either (a) that the characteristic under inspection of the pre-surgical sample can be classified as a natural variation of the post-surgical sample and in this case the comparison results in a significant similarity or (b) that the characteristic under inspection cannot be classified as such and in this case the comparison results in a significant difference. At this stage, a significant difference shows that there is a disengagement of the pre and post-op samples, since their origin could not be attributed to the same writer.

\section{Characteristics of the volunteers}

During this research six volunteers completed the post-surgical questionnaire. This number of questionnaires was extracted from a far larger pool from which the majority of volunteers were excluded by the research protocol, which is elaborated below.

Overall the patients exhibited the following characteristics:

- Out of the 6 volunteers used in the research, 3 were male and 3 female. 
- All were right-handed writers (during the first part of the examination, no ambidextrous writers appeared).

- The average age of the males were 75.3 years old and of the females 74.3 years old.

- Five volunteers possessed a below medium educational level, while one possessed an above medium educational level.

The eligibility criteria required that volunteers were free from all not controllable by them (i.e. extrinsic) factors that may influence either vision or handwriting ability. The criteria are summarised below and required that subjects:

- possessed normal and transparent cornea and anterior chamber.

- were free from any neurological and ophthalmological pathology (other than cataract) and normal binocular vision. Subjects with any prior intraocular surgical intervention were excluded.

— had lenticular cataracts grade 3 (pronounced) or 4 (severe).

— possess the below ophthalmological data: Best-corrected distant decimal visual acuity of $\leq 0.6$ in each eye, Spherical equivalent $\leq 6.00 \mathrm{D}$ in each eye, Anisometropia $<2.00 \mathrm{D}$.

This inclusion protocol guaranteed that only cases of "pure cataract" were examined. Therefore these 6 volunteers did not exhibit any condition that may influence their handwriting, except of the condition of cataractus vision, and therefore any difference found between the pre- and post-surgical samples would be attributed only to this factor.

\section{Findings}

\subsection{General Design}

There is a notable similarity between the samples in how the overall shape, and the trajectories and loops which form it are drawn and thus the general design in the pre-op samples falls within the range of natural variation of the comparison material. The forensic comparison shows no significant difference between the two sample groups of each writer. In Figure 3 certain similarities in the general design of the allographs are presented. 


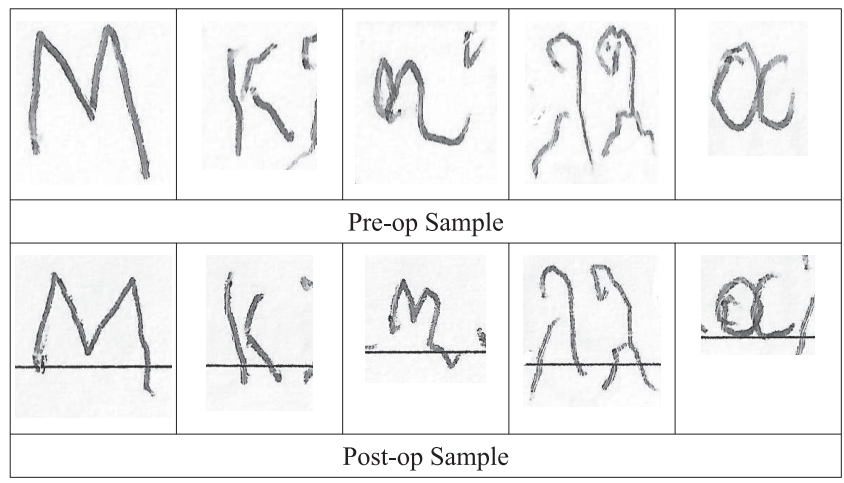

Figure 3. An example of pre-op and post-op writing samples from the same volunteer

A number of significant similarities are presented in the above Figure: The red arrows show the manner of construction of the letter $\kappa$ along with the pen lift at the center of its right part. The red circle shows the clockwise starting hook at the left part of the letter $\eta$. The blue arrow shows the pen lifts at the center of the letters $\lambda$, while the blue circle the hook at the top of the same letter - it is interesting to note that the two variations of this letter are present in both comparison groups. Lastly, the black arrow shows the starting point of the initial trajectory of the letter $\alpha$. No significant differences were found.

\subsection{Line Quality}

The post-surgical samples show higher Line Quality based on less writing tremor, a higher degree of fluidity, lower pressure and higher speed of allograph creation. This is linked to the improvement in visual feedback during the writing process during the production of the postoperative samples. An example of this is shown in Figure 4 where the red arrows pinpoint areas of writing tremor in the pre-op sample, that is localized parts of the signature where the control of the writing medium is reduced. Such effect does not appear in the post-op sample. However, the degree of the difference in the line quality is not significant (i.e. could not differentiate the origin of the two handwriting samples). 


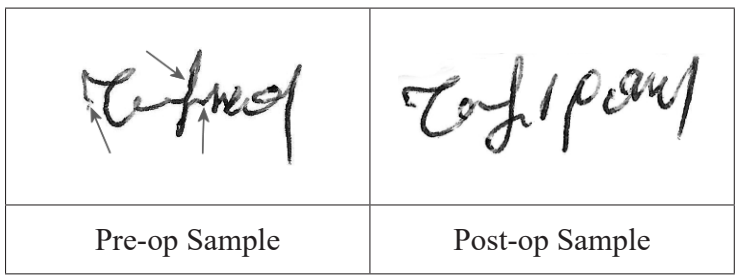

Figure 4. An illustration of the different level of Line Quality between the pre-op and post-op samples of the same writer

\subsection{Vertical and Horizontal Size}

The examination of size shows that this characteristic manifests a large mutability between the two conditions. Comparison shows that only a $16.6 \%$ of the samples retained the same horizontal size whilst changes in the Vertical Extensions were found at the $50 \%$ of the samples. Figure 5 shows such a change in both the horizontal and the vertical size in a comparison.

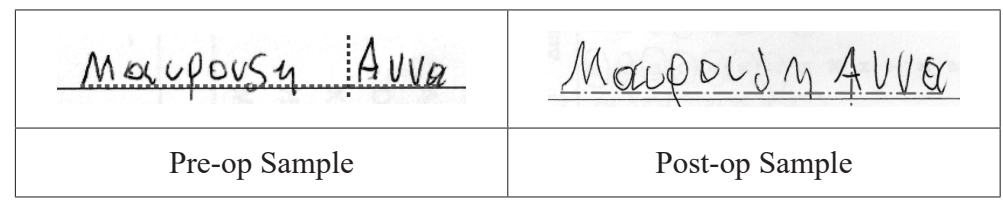

Figure 5. Both horizontal (red line) and vertical (blue line) size are enlarged in the post-op (free of cataract) sample

\subsection{Inter-allograph ratio}

The ratio of the size of each allograph to the other allographs inside the pre-op signature was measured and compared to the equivalent ratio of post-op signatures resulting in a score of significant similarity. Inter-allograph ratio scores a high percentage of similarity $(83,3 \%)$ in the comparison. Figure 6 shows examples of significant similarities of this individual characteristic. Those similarities present a high level of automation and complexity. 


\begin{tabular}{|c|c|}
\hline \multicolumn{2}{|c|}{ Significant Similarity in intra-allograph ratio } \\
\hline DEbrira THarisaun & DEGoive TMavidaun \\
\hline Pre-op Sample & Post-op Sample \\
\hline
\end{tabular}

Figure 6. A comparison of pre-op and post-op inter allograph ratios from the same volunteer

In the above figure the size of the allographs of " $\Delta$ ", " $\varepsilon$ " and " $\sigma$ " are compared in the black circular shape. The size of the allographs of " $\alpha$ " and " $v$ " are compared in the green circular shape. In the dotted black circular shape, the size of the allographs of " $K$ " and " $\eta$ " are compared. The ratio of these comparisons belong in the same variation group before and after the cataract surgery.

\subsection{Alignment}

Alignment corresponds to the positioning of the specific allographs inside the signature. This characteristic exhibits a large degree of overall constancy $(83.3 \%)$. Figure 7 shows an example of highly individualized similarity of alignment.

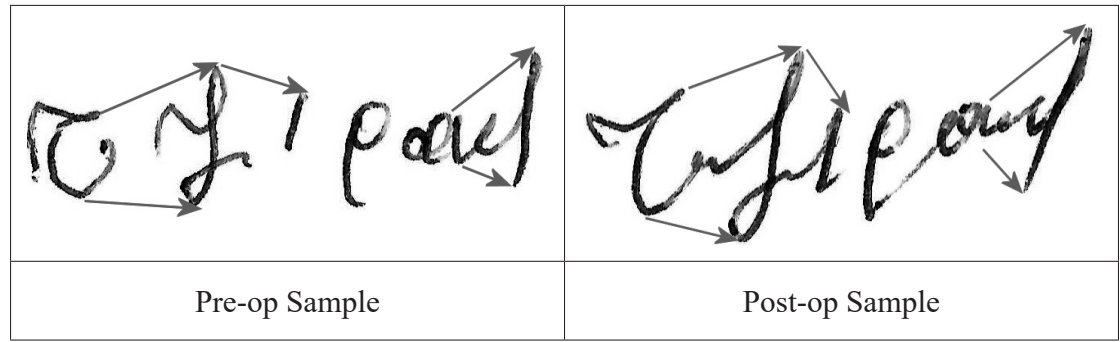

Figure 7. The red arrows point at similarities in the positioning of the corresponding allographs in pre-op and post-op signatures 


\subsection{Spacing}

Spacing includes both intra-word and inter-word spacing - furthermore features like the mixed and uniform spacing are examined here. The overall comparison of the Spacing element shows a differentiation at the $67.7 \%$ of the samples. Figure 8 shows an example of dissimilarity between spacing in the pre and post-op signatures.

\begin{tabular}{|c|c|}
\hline \multicolumn{2}{|c|}{ Significant dissimilarity in the characteristic of spacing } \\
\hline 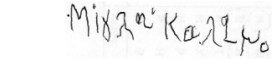 & 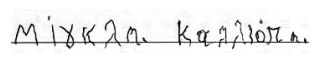 \\
\hline Pre-op Sample & Post-op Sample \\
\hline
\end{tabular}

Figure 8. The black arrows show the difference of the interword spacing between the samples

This difference by itself would not lead to an erroneous conclusion (could not be regarded as significant difference) taking into consideration the amount of consistent significant similarities of the two samples in highly individualized characteristics, mainly intra allograph ratio, alignment and general design of the allographs).

\subsection{Slant}

Slant examines the inclination of allographs relative to the perpendicular to the baseline of the writing. The analysis shows that all allographs retained the same slant in both the pre and the post operation group. In Figure 9, one such comparison is presented.

\begin{tabular}{|c|c|}
\hline \multicolumn{2}{|c|}{ Significant Similarity in the characteristic of Slant } \\
\hline Pre-op Sample & Post-op Sample \\
\hline
\end{tabular}

Figure 9. A comparison of the pre-op and post-op slant of the same volunteer 
In the above Figure the inclination of individual letters of the pre-op samples relative to the perpendicular to the baseline of the writing was measured and then compared with the inclination of the equivalent letters of the post-op samples. It must be noted that the pre-op signature shows a highly characteristic and variable group of slants, which all remain constant after the surgery.

\subsection{Summary of the findings}

In Table 1 the percentage manifestation of signs of limited visual feedback due to cataractus vision is shown.

Table 1 . The percentage of existence of signs of limited visibility in the cataractic samples

\begin{tabular}{|l|c|}
\hline Individual Characteristics & $\begin{array}{c}\text { Appearance of signs of limited visual feedback } \\
\text { in the comparison between pre } \\
\text { and post op samples }\end{array}$ \\
\hline General Design & $0,00 \%$ \\
\hline Line Quality & $83,33 \%$ \\
\hline Horizontal Extensions & $83,33 \%$ \\
\hline Vertical Extensions & $50,00 \%$ \\
\hline Inter Allograph Ratio & $16,67 \%$ \\
\hline Alignment & $16,67 \%$ \\
\hline Spacing & $66,67 \%$ \\
\hline Slant & $0,00 \%$ \\
\hline
\end{tabular}




\section{Blind trial}

The samples were summed in 6 groups - one per writer. Then each group was divided in two subgroups, one containing the pre-op samples (Subgroup A) and the second containing the post-op samples (Subgroup B). All the samples were sent to a trained FDE who was not informed that each paired group was produced by the same writer. Accordingly, the FDE was not given any detail of the nature of the research or of the medical status of the writers.

After examining the 6 groups, following the comparison methodology of the Forensic Document Examination, he correctly concluded that each subgroup A was written by the same writer who also wrote the equivalent subgroup $\mathrm{B}$, while he made no erroneous judgment as to perceive the samples of the one subgroup as an imitation attempt of the second subgroup.

\section{Discussion}

The above findings suggest that cataractus vision does not influence significantly the individual characteristics of handwriting to an extent that the pre-operational samples would appear to belong to a different variation group than that of the post-operational samples. Both the forensic comparison and the blind test furthermore suggest that the influence of cataractus vision is such that it would not lead an expert to an erroneous conclusion. Certain findings of the comparison could be used as signs of limited visual feedback: it was noted that the line quality, the size and the spacing change during the pre-op samples (the samples written under the influence of cataract), while the slant of the allographs, the inter-allograph ratio and the alignment remain constant. To sum up, visual feedback is presented not so much as a factor that interacts with the creation of the allographs, but mostly as a tool of macro-managing and inspecting the overall outcome of the combination of the above allographs and the reduced visual feedback due to cataractus opacification presents the final pictorial outcome with a limited pictorial distortion as was witnessed by the decrease of line quality or the change of the size 
and spacing — but its degree and extent is so localized and minimal that it cannot influence the individual characteristics to a degree that can create a differentiation between the two subgroups.

\section{References}

Abdelghany, A.A. and Alio, J.L., "Surgical options for correction of refractive error following cataract surgery", Eye and Vision, vol. 1(2), 2014.

Cunningham, E.T. and Riordan-Eva, P., Vaughan \& Asbury's General Ophthalmology. (18th ed.). McGraw-Hill Medical, 2011.

Duker, M. and Yanoff, J.S. Ophthalmology ( $3^{\text {rd }}$ Ed.) Mosby, Edinburgh 2008.

Hodge, W.G., Whitcher, J.P. and Satariano, W. "Risk factors for age-related cataracts", Epidemiologic Reviews, vol. 17(2), 1995, pp. 336-346.

Jaffe, N.S., Cataract Surgery and Its Complications. $5^{\text {th }}$ ed., St Louis, Mosby Year Book, 1989.

Kador, P.F., "Overview of the current attempts toward the medical treatment of cataract", Opthalmology, vol. 90 (4), pp. 52-364, April 1983.

Livingston, P.M. Carson, C.A. and Taylor, H.R., "The epidemiology of cataract: a review of the literature", Ophthalmic Epidemiol. vol. 2(3), pp. 151-164, Dec 1995.

Meadow, N.B., "Cryotherapy: A fall from grace, but not a crash", Ophthalmology Times, vol. 30, Issue 20, p. 66, October 15, 2005.

Mojon-Azzi, S.M. and Mojon, D.S., "The rate of outpatient cataract surgery in ten European countries: an analysis using data from the SHARE survey", Opthalmologie, vol. 245(7), pp. 1041-1044, July 2006.

D’Ocampo, M.V.V., Senile Cataract, Cataract Surgery and its Complications, St Osborn, Albert S. Questioned Documents, Albany, Boyd Printing Co, 1929.

Pavan-Langston, D., Manual of Ocular Diagnosis and Therapy, Little, Brown and Company, 1990.

Slamovits, T.L., Lens and Cataract. Section 11, American Academy of Opthalmology, San Francisco 1993.

Sliney, D.H., "UV radiation ocular exposure dosimetry". Doc. Ophthalmol, vol. 88(3-4), pp. 243-254, March 1994.

Smith, G.T. and Liu, C.S.C., Is it time for a new attitude to "simultaneous" bilateral cataract surgery, Br.J., Opthalmology, vol. 85(12), pp. 1489-1496, 2001.

Yamaguchi, T. Negishi, K. and Tsubota, K., "Functional visual acuity measurement in cataract and intraocular lens implantation", Current Opinion in Optamology, vol. 22(1), pp. 31-36, January 2011.

Yanoff, M. and Duker, J.S., Ophthalmology ( ${ }^{\text {rd }}$ ed.), Edinburgh: Mosby 2008.

The picture in Figure 1 is property of www.simpleeyes.org.

The picture in Figure 2 is property of Rakesh Ajuja, MD. 


\section{Summary}

The effects of cataractus vision on handwriting performance are evaluated. Six cataract patients were enrolled with an average age of $74.8 \mathrm{yrs}$ and completed a specially designed questionnaire with samples of their signature pre-operatively and three-months post-operatively. The signatures were analyzed through a four-eye principle peer reviewed examination focusing on six basic general characteristics of writing (line quality, general design, size, arrangements, spacing and slant) and their sub-elements. The qualitative analysis showed that cataractus vision causes a decrease of line quality and differences between the pre- and post- surgical samples on the sub-element of size. General design, intra-allograph ratio and slant were not influenced by the compromised vision.

Keywords: handwriting, signature, cataract vision, visual feedback, pictorial characteristics. 